Instituto Internacional de Investigación y Desarrollo Tecnológico Educativo INDTEC, C.A.

DOI: https://doi.org/10.29394/Scientific.issn.2542-2987.2021.6.22.4.78-95

OAI-PMH: http://www.indteca.com/ojs/index.php/Revista Scientific/oai

Artículo Original / Original Article

\title{
Análisis de prevención en las drogodependencias en el barrio Jaime Roldós Aguilera, Quito
}

\author{
Autores: Eduardo Efraín Calero Brito \\ Universidad Internacional SEK, UISEK \\ eecalero.mpp@uisek.edu.ec \\ Quito, Ecuador \\ https://orcid.org/0000-0002-3441-7774 \\ Jhon Alberto Ramírez Baldeón \\ Universidad Internacional SEK, UISEK \\ jaramirez.mpp@uisek.edu.ec \\ Quito, Ecuador \\ https://orcid.org/0000-0002-7994-2281
}

\section{Resumen}

Existen modelos de prevención en el ámbito de drogodependencias desde un enfoque comunitario, mismos que han mostrado eficacia en la reducción de niveles de consumo. En Latinoamérica y en el Ecuador se trabaja en programas de información y concientización sobre los efectos de las sustancias psicoactivas. Sin embargo, se evidencia la ausencia de un programa específico de prevención desde una visión comunitaria. La presente investigación tiene por objeto analizar los procesos de prevención en drogodependencias en un sector de Quito, Ecuador. Se utilizó un diseño no experimental, un análisis bibliográfico documental y una metodología de tipo cualitativa a través de la cual se aplicó una entrevista semiestructurada a 20 líderes comunitarios, con el fin de obtener su percepción con relación a la temática. Posterior al análisis se ha encontrado que el $85 \%$ de los encuestados considera que existe un problema de consumo, y el $70 \%$ manifiesta que no cuenta con estrategias de manejo o detección oportuna de los casos de consumo. Por esta razón concluimos que la comunidad estudiada no cuenta con un programa de prevención de drogodependencias y que existe la presencia de un problema de consumo de drogas.

Palabras clave: adicción; consumo; estupefaciente; prevención.

Código de clasificación internacional: 6113.05 - Tratamiento de la drogadicción.

Cómo citar este artículo:

Calero, E., \& Ramírez, J. (2021). Análisis de prevención en las drogodependencias en el barrio Jaime Roldós Aguilera, Quito. Revista Scientific, 6(22), 78-95. Recuperado de: https://doi.org/10.29394/Scientific.issn.2542-2987.2021.6.22.4.78-95

Fecha de Recepción: 12-01-2021
Fecha de Aceptación: 03-06-2021
Fecha de Publicación: 05-11-2021 
OAI-PMH: http://www.indteca.com/ojs/index.php/Revista Scientific/oai

\title{
Analysis of prevention in drug addiction in the Jaime Roldós Aguilera neighborhood, Quito
}

\begin{abstract}
There are prevention models in the field of drug addiction from a community approach, which have shown efficacy in reducing consumption levels. In Latin America and Ecuador, information and awareness programs are being carried out on the effects of psychoactive substances. However, the absence of a specific prevention program from a community perspective is evident. The present research aims to analyze the prevention processes in drug addiction in a sector of Quito, Ecuador. A non-experimental design, a documentary bibliographic analysis and a qualitative methodology were used through which a semi-structured interview was applied to 20 community leaders, in order to obtain their perception regarding the issue. After the analysis, it has been found that $85 \%$ of those surveyed consider that there is a problem of consumption, and $70 \%$ state that they do not have strategies for the management or timely detection of cases of consumption. For this reason, we conclude that the studied community does not have a drug addiction prevention program and that there is a problem of drug use.
\end{abstract}

Keywords: addiction; consumption; narcotic; prevention. International classification code: 6113.05 - Drug therapy.

\section{How to cite this article:}

Calero, E., \& Ramírez, J. (2021). Analysis of prevention in drug addiction in the Jaime Roldós Aguilera neighborhood, Quito. Revista Scientific, 6(22), 78-95, e-ISSN: 2542-2987. Recovered from: https://doi.org/10.29394/Scientific.issn.2542-2987.2021.6.22.4.78-95

Date Received: 12-01-2021
Date Acceptance: 03-06-2021
Date Publication: 05-11-2021 


\section{Introducción}

El consumo de drogas y alcohol ha sido un tema de interés mundial, desde hace décadas, debido a los efectos negativos que produce la exposición, experimentación y habitación a las sustancias, considerando esta premisa se han desarrollado programas de tratamiento desde diferentes enfoques y en diversas modalidades. En el año 2015 la Oficina de las Naciones Unidas contra la droga y el delito emite un informe menciona que 28 millones de personas han desarrollado drogodependencias, es decir, que han generado un vínculo con varias sustancias.

La Oficina de las Naciones Unidas contra la Drogas y el Delito (UNODC, 2015): menciona en su informe la innegable necesidad de crear políticas públicas dirigidas a prevenir y a intervenir en las drogodependencias, exponiendo como un respaldo de la necesidad las siguientes cifras: cerca de 14 millones de personas tienen adicción a drogas de tipo inyectables y aproximadamente 1.5 millones han contraído el Virus de la inmunodeficiencia humana (VIH), por lo que se han levantado el llamado para brindar una oportuna atención

La creación de políticas públicas debe responder a la necesidad actual de la población a quien va a ir dirigido los programas de prevención, según el Observatorio Europeo de las Drogas y las Toxicomanías (EMCDDA, 2017): se debe considerar factores de tipo sociodemográfico como la edad, sexo, salud física, salud mental, influencias biológicas, situación económica, factores familiares, etnia, religión y orientación sexual.

Al ser un problema de origen multifactorial, se debe dar también un enfoque dinámico de abordaje. En este sentido, Díaz y Amaya (2012): proponen considerar los componentes ambientales e individuales en el consumo de sustancias legales o ilegales, relacionándose estrechamente con elementos de la personalidad.

Señalando a Martínez y Arana (2015a): la perspectiva de la cultura 
latinoamericana que tienen sobre las sustancias psicoactivas han normalizado su consumo, la misma que conlleva tener una visión del mundo de las drogas basada en experiencias internas, mantenerse en este discurso puede impedir evaluar los efectos negativos de las sustancias en los contextos de relación social, si una cultura permite el consumo, se dará paso a componentes sociales de permisividad y libre acceso, y podrían desencadenar en una sociedad que alienta el consumo, situación que contrasta con países como Portugal.

En Portugal se han descrito Políticas sobre Drogas, un análisis de los beneficios de la despenalización del consumo, mismas que surgieron en base a un exhaustivo análisis de resultados de investigaciones en ese país desde el año 2001, donde se evidenció un consumo elevado de alcohol y otras drogas, el Ministerio de Salud de Portugal diseño un plan de tratamiento preventivo desde lo comunitario, con acciones encaminadas a la difusión de información, gestión de recursos y campañas en territorio Portugués, para Domosławski (2012): la experiencia de este país ha generado gran impacto a nivel mundial por los resultados conseguidos a la actualidad, con la reducción de sus índices de consumo, han sido replicados en países de la Unión Europea.

En el año del 2001, fue Portugal quien propuso la despenalización del consumo de drogas ilegales, incluyendo la cocaína y heroína, aspecto que no tenía precedentes en su incidencia. Sin embargo, la modificación en las leyes en temas relacionados con drogas significó un hito histórico en Portugal. Martínez y Arana (2015b): mencionan por su parte la despenalización sociocultural que tuvo por efecto dicho proceso, consiguiendo en particular que el consumo de sustancias deje de abordarse como un hecho de delito y que se considere más bien como un problema de salud pública que requiere una atención prioritaria. 
Esta idea se aparenta con lo propuesto por Beck, Wrigth, Newman y Liese (1999): quienes mencionan la importancia de un abordaje terapéutico de las adicciones desde un modelo cognitivo realizando un análisis funcional de factores asociados al consumo y componentes de pensamiento que afectan la funcionalidad de la vida de las personas con consumo de sustancias. Para Soto (2013): han surgido nuevas conductas adictivas que merecen una atención desde lo terapéutico, pero se debe enfocar en primer lugar la promoción y educción sobre temas relacionados al consumo.

En España de igual forma se ha descrito la realidad del problema de consumo de sustancias, teniendo como población efecto de estudio a adolescentes, que presentan mayores componentes de contingencia que se asocian a la exposición de consumo de sustancias, en este mismo orden de ideas, como señalan Orte y Ballester (2018): adicionalmente se habla sobre la efectividad de un programa de prevención intervención familiar, mismo que tiene como objetivo fomentar las habilidades parentales, la comunicación dentro de la familia, mejoras en las habilidades prosociales de los adolescentes, con el fin de disminuir los riesgos y potenciar la conducta prosocial, este enforque de tipo preventivo ha sido eficaz, además ha sido utilizado en adolescentes con conductas problemáticas y comportamientos disociales.

De igual forma en un análisis en prevención del consumo de sustancias psicoactivas en niños niñas y adolescentes en España y Latinoamérica se ha descrito como factor primordial que las acciones que están direccionadas al cambio actitudinal con los aspectos de consumo de sustancias legales e ilegales además la vinculación comunitaria, en conformidad con Castaño, Bocanegra y Gantiva (2017): es decir, la participación familiar y de entidades educativas, desempeñan un rol proactivo y generan factores protectores frente al consumo de sustancias a diferencia de aquellos programas que solo constan de información proporcionada mediante charlas, no tienen efectividad, 
se concluyó que el fomento de habilidades para la vida, los valores y el empleo del tiempo en actividades productivas.

En ese marco, Ochoa y Madoz (2008): en su investigación realizada en Madrid España sobre los riesgos laborales y factores relacionados al consumo de drogas, argumentan que existen porcentajes elevados de personas que desempeñan una actividad laboral, presentan problemas moderados y graves de consumo de sustancias, por lo que se estima necesario la creación e implementación de políticas laborales y medidas preventivas que estén encaminadas a reducir los riesgos psicosociales del consumo de drogas.

Paralelamente, Hernández (2015): quien realizó una evaluación sobre la efectividad del programa de prevención de sustancias denominado YOMIVIDA en Colombia, mismo que estaba enfocada a población menor de 14 años, se evaluaron las aptitudes para la vida como factores protectores para la exposición al consumo de drogas, participaron un estimado de 42 unidades educativas, que aplicaron este modelo, se encontró que los docentes reconocer que es importante un acompañamiento directo con los estudiantes y trabajar en conjunto con los padres, quienes potencias las habilidades para la vida, adicionalmente se encontró que el alcohol, tabaco y marihuana se consume en edades escolares, y se destacó el rol primordial de la escuela frente a la prevención.

Para autores como Nieto-Ortiz y Nieto-Mendoza (2020): quienes hablan sobre la importancia de un estudio en Colombia sobre el ambiente, los lugares y sitios que fomentan el consumo, posterior al análisis se ha determinado que un $43.9 \%$ de los encuestados revelan un consumo de alcohol como sustancias de mayor elección y de exposición frecuente y que guarda relación con el formar parte de un ambiente universitario con la presencia de consumo de tabaco en un $100 \%$ y marihuana en un $74 \%$, como factor común en quienes están expuestos a estos niveles de consumo se encuentra que; utilizan el tiempo de ocio en frecuentar lugares como bares, rumbas y discotecas, por lo 
que se describe la importancia de un plan de enfoque preventivo que este enfocado en la comunidad.

En el contexto ecuatoriano de acuerdo con Naranjo y Jaramillo (2017): en su informe da a conocer el costo para el país desde la visión económica de afrontar los problemas relacionados con las drogas y su consumo, haciendo un especial énfasis en la inversión estatal, la participación de entidades gubernamentales públicas y privadas para reducir y minimizar los efectos del consumo de sustancias, por lo que el trabajo desde la promoción y prevención es de vital importancia.

De igual forma en Ecuador para los autores Barreno, Pazmiño e Iriarte (2020): quienes realizaron una investigación en población militar en el evaluando la predisposición frente al consumo de sustancias en 1118 personas, encontraron que cerca de 885 aspirantes tenía una percepción adecuada sobre las implicaciones de consumir drogas, que se relacionan con la actitud de los aspirante a no exponerse al consumo, adicionalmente se halló que uno de los factores protectores podría ser identificarse con alguna creencia religiosa, por lo que se concluyó que es importante el diseño de programas preventivos de enfoque comunitario, con alcance familiar.

Así mismo, el Ministerio de Salud Pública ha realizado una investigación, dirigida estudiantes de educación básica y bachillerato, en la que se pudo evidenciar que el cantón Quito, Ecuador, ocupa el octavo lugar por encima de la media nacional en consumo de drogas ilícitas, es decir, que en el Ecuador existe un consumo elevado de sustancias en los jóvenes de 12 y 18 años.

El Ecuador cuenta con un Proyecto en Prevención y Control Socio Económico de Drogas 2017-2021, mismo que recibió la aprobación de la Organización de los Estados Americanos; Comisión Interamericana para el Control del Abuso de Drogas (OEA-CICAD, 2019): dicho programa está basado en los siguientes ejes: fortalecimiento en conocimiento y recursos de 
las entidades gubernamentales, disminución de la oferta, demanda, e implementación de normas regulación y subvención internacional, además consta de una evaluación y actualización para determinar la eficacia del plan de acción, teniendo como actores sociales a los principales Ministerios de Gobierno, Salud, Educación, Deporte, entidades académicas dedicadas a la investigación.

Por lo que es importante realizar un análisis metódico de la información en relación a la creación de programas preventivos, considerando que las políticas públicas de países latinoamericanos no satisfacen la necesidad y no se ajustan a la realidad del consumo de sustancias que va en aumento, tomando en cuenta la eficacia de los programas preventivos, para Pérez, Mejía y Becoña (2015): estos deben tener una estructura que consiste en un análisis, evaluación y seguimiento para así saber si es viable aplicarlo dentro de un contexto especifico.

Se entiende como prevención a la suma de estrategias que se toman con antelación previa, antes que suceda una determinada acción y de esta forma lograr reducir los efectos negativos, dentro del ámbito de adicciones o drogodependencias, a juzgar por la Comisión Nacional para el Desarrollo y Vida sin Drogas (DEVIDA, 2015a): la prevención se basa en la identificación oportuna de aquellos factores asociados que favoreces el inicio de la conducta de exposición a sustancias, además de analizar los efectos que podrían resultar de esta acción.

La Comisión Nacional para el Desarrollo y Vida sin Drogas (DEVIDA, 2015b): expone que la prevención se desarrolla a través de un conglomerado de actividades planificadas y destinadas a reducir y limitar los factores que dan paso al uso de sustancias psicoactivas, fomentando los factores de ayuda, además de la seguridad dentro del núcleo familiar, el crecimiento de las capacidades de afrontamiento y de alternativas viables para que el medio comunitario, social, académico y familiar actúan de forma coordinada para 
conseguir los resultados esperados.

El objetivo del presente estudio es demostrar la importancia de tratar el consumo problemático de sustancias desde una perspectiva que tome en cuenta al individuo como ente social, desde los componentes emocionales y psicológicos que configuran su personalidad y que afectan a la comunidad en la que este individuo se inscribe.

\section{Metodología (Materiales y métodos)}

Para los fines del presente estudio se utilizó la metodología bibliográfica-documental y desde un enfoque cualitativo de investigación. La recolección de datos bibliográficos (informes, artículos científicos y protocolos) abarcó un análisis del estado de la cuestión en países como España, Portugal, Colombia y Ecuador.

Con el fin de conocer el alcance que podría tener el diseño de programas comunitarios de prevención del consumo de drogas, se realizó una encuesta de 8 ítems a 20 líderes comunitarios y familiares de la Cooperativa de vivienda Jaime Roldós Aguilera en la ciudad de Quito, Ecuador.

Para la selección de la muestra se consideraron los siguientes criterios de inclusión: ser morador de la Cooperativa de vivienda, ser líder familiar de opinión comunitaria y suscribir un consentimiento informado. Los criterios de exclusión fueron moradores en desacuerdo de participar y no ser considerados líderes familiares por parte de la opinión comunitaria.

Previo a la aplicación de la encuesta, se realizó un trabajo de inmersión en la comunidad, mediante la observación y la participación de los investigadores en diferentes espacios de la vida en comunidad. A partir de este contacto, se obtuvo la autorización de los líderes comunitarios para aplicar la encuesta. 


\section{Resultados (análisis e interpretación de los resultados)}

A continuación, se describen los resultados de la encuesta, detallándose los 8 ítems que constituían la encuesta diseñada.

Tabla 1. Tipos de drogas que conoce.

\begin{tabular}{|l|c|}
\hline Número de drogas & Porcentaje de población \\
\hline Ninguna & $65 \%$ \\
\hline 1 a 5 & $30 \%$ \\
\hline Más de 6 & $5 \%$ \\
\hline
\end{tabular}

Fuente: Los Autores (2020).

En la tabla 1 se evidencia que el $65 \%$ de los encuestados refieren que no tienen conocimiento sobre ningún tipo de drogas, el $30 \%$ distinguen entre 1 a 5 tipos de drogas, mientras que un $5 \%$ coincide que conoce más de 6 clases de drogas, por lo que se infiere que la mayor parte de los líderes comunitarios desconocen sobre las sustancias.

Tabla 2. ¿Ha consumido drogas alguna vez?

\begin{tabular}{|l|c|}
\hline Opciones de respuesta & Porcentaje de población \\
\hline SI & $65 \%$ \\
\hline NO & $35 \%$ \\
\hline Total & $100 \%$ \\
\hline
\end{tabular}

Fuente: Los Autores (2020).

En la tabla 2, se evidencia que el $65 \%$ de los encuestados ha consumido drogas en algún momento de su vida, y en menor proporción, un 35\% no lo ha hecho por lo que se podría inferir que existe un alto nivel de riesgo de factores comunitarios que podrían favorecer el aparecimiento de conductas relacionadas al consumo de drogas.

Tabla 3. ¿Considera que existen drogas legales?

Fuente: Los Autores (2020)

\begin{tabular}{|l|c|}
\hline Opciones de respuesta & Porcentaje de población \\
\hline SI & $20 \%$ \\
\hline NO & $80 \%$ \\
\hline Total & $100 \%$ \\
\hline
\end{tabular}


En la tabla 3, se evidencia que la mayoría de los encuestados representado por el $80 \%$ no considera que existan drogas legales, mientras que el $20 \%$ piensa que sí, lo que nos anima a pensar que aún se presenta el estigma social en relación con las drogar, mismo que podría condicionar las actitudes frente al consumo.

Tabla 4. ¿Cuáles son las drogas de mayor consumo?

\begin{tabular}{|l|c|}
\hline Opciones de respuesta & Porcentaje de población \\
\hline Tabaco & $10 \%$ \\
\hline Marihuana & $30 \%$ \\
\hline Alcohol & $60 \%$ \\
\hline Total & $100 \%$ \\
\hline
\end{tabular}

Fuente: Los Autores (2020).

En la tabla 4, se evidencia que la droga de mayor consumo en la Cooperativa Jaime Roldós Aguilera según la perspectiva de los líderes comunitarios y familiares es el alcohol con un $60 \%$, seguida de la marihuana con un $30 \%$ y finalmente en menor proporción tabaco con $10 \%$, datos que coinciden con lo descrito en las investigaciones, que revelan que en Ecuador encabeza la lista de consumo de alcohol.

Tabla 5. ¿Qué consecuencias tiene el consumo de drogas en las personas?

\begin{tabular}{|l|c|}
\hline Opciones de respuesta & Porcentaje de población \\
\hline a. Conductas violentas & $35 \%$ \\
\hline b. Muerte & $30 \%$ \\
\hline c. Sobredosis & $25 \%$ \\
\hline d. Trastorno mental & $10 \%$ \\
\hline Total & $100 \%$ \\
\hline
\end{tabular}

Fuente: Los Autores (2020).

La tabla 5, revela que la mayoría de los encuestados, $35 \%$ consideran que la consecuencia más conocida del consumo de sustancias es la presencia de conductas violentas. Le sigue el riesgo de muerte $30 \%$ y sobredosis $25 \%$. Finalmente, sólo un porcentaje menor de los encuestados el 10\% consideran que el consumo puede acarrear trastornos mentales. De manera general, la 
percepción relacionada con el consumo de sustancias es el de perjuicio para la salud y para la vida.

Tabla 6. ¿Presión a una persona a consumir drogas?

\begin{tabular}{|l|c|}
\hline Opciones de respuesta & Porcentaje de población \\
\hline Amigos & $40 \%$ \\
\hline Familia & $35 \%$ \\
\hline Medios Televisivos & $25 \%$ \\
\hline Total & $100 \%$ \\
\hline
\end{tabular}

Fuente: Los Autores (2020).

En la Tabla 6, se evidencia que el $40 \%$ de los encuestados concuerda en que los amigos son quienes ejercen presión para iniciar un consumo de drogas, el $35 \%$ refiere que es la familia, mientras que el $25 \%$ afirma que son los medios televisivos. Lo que indica que el factor social y la ausencia de habilidades de toma de decisiones de forma autónoma podrían desencadenas en el inicio de consumo de drogas.

Tabla 7. ¿Existe un problema de consumo en su cooperativa?

\begin{tabular}{|l|c|}
\hline Opciones de respuesta & Porcentaje de población \\
\hline SI & $85 \%$ \\
\hline NO & $15 \%$ \\
\hline Total & $100 \%$ \\
\hline
\end{tabular}

Fuente: Los Autores (2020).

De acuerdo con la tabla 7 , el $85 \%$ del total de los encuestados manifiesta que, si existe un problema de consumo en la cooperativa Jaime Roldós Aguilera. Apenas el 15\% ignora o no reconoce la problemática.

Tabla 8. ¿Conoce sobre estrategias de detección y manejo de problemas de consumo de drogas?

\begin{tabular}{|l|c|}
\hline Opciones de respuesta & Porcentaje de población \\
\hline SI & $20 \%$ \\
\hline NO & $70 \%$ \\
\hline TAL VEZ & $10 \%$ \\
\hline Total & $100 \%$ \\
\hline
\end{tabular}

Fuente: Los Autores (2020). 
Finalmente, en la tabla 8, se evidencia que la mayor parte de los líderes comunitarios encuestados $70 \%$ refieren que no conocen sobre las estrategias o herramientas de detección y manejo de problemas relacionados al consumo de drogas. Apenas el $20 \%$ dice conocer estas estrategias de manera general, y el $10 \%$ se pronuncia con relación a su conocimiento relativo sobre el tema.

\section{Conclusiones}

Los resultados revelan que existe un índice alto de consumo de sustancias psicoactivas en la cooperativa de vivienda Jaime Roldós Aguilera índice que estaría relacionado con factores de tipo social, familiar y componentes individuales que desencadenan el inicio y exposición al uso y abuso de sustancias psicoactivas; considerando además los factores de tipo individual y rasgos de personalidad.

Concluimos además que los moradores de la cooperativa de vivienda Jaime Roldós Aguilera, desconocen sobre temas relacionados a las drogodependencias y los factores de riesgo, además según los resultados de la encuesta el consumo se relaciona con la aparición de conductas violentas, demostrándose así la relación directa entre las conductas disruptivas y violentas en la adolescencia frente a la exposición en especial de consumo de cocaína y heroína, se analizaron además factores de riesgo como la permisividad de la población cercana a los sitios de consumo.

En base a los resultados se debería crear una guía preventiva para atender la problemática de las drogodependencias que tiene la cooperativa de vivienda Jaime Roldós Aguilera, en ambientes juveniles y centros de enseñanza, constituyendo un aporte teórico y práctico en los distintos sectores que se los quiera implementar. El consumo de alcohol y drogas es un problema que genera desocupación de los jóvenes, por lo que sugiere que la atención se encamine a diseñar actividades que se involucre su participación.

De acuerdo con los hallazgos a través de la encuesta, el $70 \%$ de los 
encuestados refiere desconoce sobre estrategias o herramientas de detección y manejo de la problemática de consumo de drogas, por lo que la guía debería tener una estructura que contemple componentes con las siguientes temáticas: Drogas y Adicciones, patrones de consumo, la adolescencia, factores de riesgo y protección, intervención temprana del consumo de sustancias, para la cooperativa de vivienda Jaime Roldós Aguilera.

\section{Referencias}

Barreno, J., Pazmiño, C., \& Iriarte, L. (2020). Predisposición al consumo de sustancias en aspirantes a la carrera militar. Revista Scientific, 5(Ed. Esp.), 205-225, e-ISSN: 2542-2987. Recuperado de: https://doi.org/10.29394/Scientific.issn.2542-2987.2020.5.E.10.205$\underline{225}$

Beck, A., Wrigth, F., Newman, C., \& Liese, B. (1999). Terapia cognitiva de las drogodependencias. 1ra edición, ISBN: 978-84-493-3563-1.

Castaño, L., Bocanegra, K., \& Gantiva, C. (2017). Prevención del consumo de sustancias psicoactivas en niños, niñas y adolescentes: compilación de investigaciones publicadas entre 2002 y 2015 en España y Latinoamérica. Artículo de grado. Colombia: Universidad Católica de Pereira. Recuperado de: http://hdl.handle.net/10785/4550 DEVIDA (2015a,b). Manual para la prevención de consumo de Drogas. San Isidro, Depósito Legal: 1501012003-5241. Perú: Programa de Capacitación Laboral CAPLAB; Comisión Nacional para el Desarrollo y Vida sin Drogas - DEVIDA.

Díaz, K., \& Amaya, M. (2012). Factores familiares, individuales y ambientales en el consumo y no consumo de drogas en adolescentes. Avances en Enfermería, 30(3), 37-59, e-ISSN: 25422987. Recuperado de:

https://revistas.unal.edu.co/index.php/avenferm/article/view/39862 
Domosławski, A. (2012). Políticas sobre Drogas en Portugal: Beneficios de la Descriminalización del Consumo de Drogas. ISBN: 978-1936133-82-6. New York, Estados Unidos: Fundaciones Open Society. EMCDDA (2017). Health and social responses to drug problems. ISBN: 978-92-9497-197-5. Lisbon, Portugal: European Monitoring Centre for Drugs and Drug Addiction.

Hernández, E. (2015). Evaluación de un programa de prevención del consumo de sustancias psicoactivas para la infancia. Salud y drogas, 15(1), 67-77, e-ISSN: 1578-5319. Recuperado de: https://www.redalyc.org/articulo.oa?id=83938758007

Martínez, D., \& Arana, X. (2015a,b). ¿Qué es la normalización en el ámbito de los usos de las drogas?. Revista Española de Drogodependencias, 40(3), 27-42, e-ISSN: 0213-7615. Recuperado de:

https://www.aesed.com/upload/files/vol-40/n-3/v40n3 2.pdf

Naranjo, F., \& Jaramillo, D. (2017). Costo del fenómeno de las drogas en Ecuador en 2015. Quito, Ecuador: Secretaria Técnica de Drogas.

Nieto-Ortiz, D., \& Nieto-Mendoza, I. (2020). Consumo de sustancias psicoactivas: un estudio descriptivo-transversal en la Universidad del Atlántico. Revista de Psicología Universidad de Antioquia, 12(1), 7-24, e-ISSN: 2145-4892. Recuperado de:

https://doi.org/10.17533/udea.rp.v12n1a01

Ochoa, E., \& Madoz, A. (2008). Consumo de alcohol y otras drogas en el medio laboral. Medicina y seguridad del trabajo, 54(213), 25-32, eISSN: 1989-7790. Recuperado de:

https://dialnet.unirioja.es/servlet/articulo?codigo $=2885751$

OEA-CICAD (2019). Mecanismo de Evaluación Multilateral (MEM). ISBN: 978-0-8270-6888-9. Ecuador: Organización de los Estados Americanos (OEA); Comisión Interamericana para el Control del Abuso de Drogas (CICAD). 
Orte, C., \& Ballester, L. (eds.). (2018). Intervenciones efectivas en prevención familiar de drogas. 1ra edición, ISBN: 978-84-17667-05-

4. Barcelona, España: Ediciones Octaedro, S.L.

Pérez, A., Mejía, J., \& Becoña, E. (2015). De la prevención y otras historias: Historia y evolución de la prevención del consumo de alcohol y drogas en América Latina y en Europa. ISBN: 978-958-58268-3-0. Bogotá, Colombia: California-Edit.

Soto, A. (2013). Las nuevas Adicciones ¿Qué son? ¿Cómo afrontarlas?. 1ra edición, ISBN: 978-84-92892-15-0. España: Mestas Ediciones, S.L. UNODC (2015). Informe Mundial sobre las drogas. Resumen Ejecutivo. Wdr15 Exsum S. Viena, Austria: Oficina de las Naciones Unidas contra la Drogas y el Delito. 
OAI-PMH: http://www.indteca.com/ojs/index.php/Revista Scientific/oai

Artículo Original / Original Article

\section{Eduardo Efraín Calero Brito}

e-mail: eecalero.mpp@uisek.edu.ec

Nacido en Guaranda, Ecuador el 14 de octubre del año

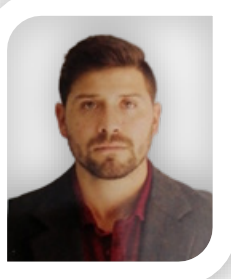
1991. Psicológico Clínico, Máster en Psicología Clínica con mención en Psicoterapia por la Universidad Internacional SEK (UISEK); Experiencia Clínica en Psicoterapia Individual, grupal y familiar, Organización No Gubernamental (ONG), Ministerio de Justicia, Superintendencia de Bancos; Departamento de Consejería Estudiantil; además participo en el desarrollo de planes de prevención en el ámbito de consumo de sustancias. 
OAI-PMH: http://www.indteca.com/ojs/index.php/Revista Scientific/oai

Artículo Original / Original Article

\section{Jhon Alberto Ramírez Baldeón}

e-mail: jaramirez.mpp@uisek.edu.ec

Nacido en San Antonio de Bayushig, Riobamba, Ecuador

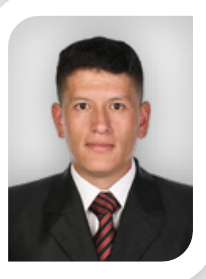

el 12 de julio del año 1992. Psicológico Clínico; Máster en

Psicología Clínica con mención en Psicoterapia por la Universidad Internacional SEK (UISEK); Experiencia

Clínica en atención ambulatoria en adicciones; poseo experiencia en el ámbito preventivo; fundador del consultorio psicológico "Pensarte"; Ministerio de salud Pública.

El contenido de este manuscrito se difunde bajo una Licencia de Creative Commons ReconocimientoNoComercial-Compartirlgual 4.0 Internacional 